Artículo 6

\title{
LA APLICACIÓN DE LOS SIG COMO HERRAMIENTA DE ANÁ- LISIS EN LA GESTIÓN DEL TERRITORIO
}

\author{
-Scognamillo Amelia - Romero Mirta. \\ Instituto Regional de Planeamiento y Hábitat. Facultad de Arquitectura, Urbanismo y Diseño. UNSJWAv. \\ Ignacio de la Roza 590 Oeste. Complejo Universitario "Islas Malvinas" _CP: 5400 - Rivadavia - San Juan. \\ Tel. 0264-4233259 e-mail: arqame@gmail.com
}

Palabras clave: SIG, Análisis, Territorio.

\section{Resumen:}

Los Sistemas de Información Geográfica (SIG) constituyen una moderna herramienta en el ámbito del manejo integral de información espacial. No obstante se trata de una tecnología todavia inmadura por sus caracteristicas complejas, que se traduce en el desconocimiento de sus potencialidades por quienes no son técnicos ni especialistas en la materia. El presente trabajo tiene por objeto reflexionar sobre las potencialidades que presentan los SIG en la gestión del territorio, cuando son utilizados como herramienta capaz de construir nuevos datos y nuevos elementos a partir de los ya existentes, en base a su análisis y procesamiento. Se toma como ejemplo para este estudio, el empleo de esta herramienta en la simulación de escenarios de riesgo sísmico en el área metropolitana de la ciudad de San Juan (AM GSJ). Se concluye el trabajo con una sintesis de las diversas posibilidades que presentan los SIG en relación a operaciones orientadas tanto a los atributos como a las entidades, con el fin de obtener tablas de datos o mapas derivados, que simulen situaciones reales o hipotéticas, relacionadas a la gestión del territorio.

\section{Introducción:}

Los Sistemas de Información Geográfica (SIG) constituyen una moderna herramienta en el ámbito del manejo integral de información espacial. Desde sus inicios alrededor de 1960 se expandieron rápidamente; y hoy sus prestaciones son permanentemente actualizadas para dar respuestas con mayor eficacia a las nuevas necesidades que el mercado le impone.
Estos programas han significado una verdadera revolución conceptual y práctica en el manejo y análisis de la información geográfica. De hecho, según CHORLEY (1987) podemos asegurar que los SIG son el paso adelante más importante desde la invención del mapa en cuanto al manejo de datos espaciales.

Una multitud de autores durante estos más de cincuenta años han definido de una manera u otra esta nueva herramienta que, apoyándose en el desarrollo de la informática, nos ayuda básicamente al tratamientos de datos del mundo en el que vivimos. No obstante la mayoría coincide en que la principal caracteristica de un SIG es el vínculo que establece entre datos alfanuméricos, que describen y caracterizan atributos de un elemento y que son almacenados en bases de datos, con los correspondientes elementos gráficos que se encuentran localizados espacialmente y a quienes se les atribuyen características particulares. Es a partir de estos vínculos que se pueden generar numerosas y diversas operaciones y/o consultas con el objeto de concebir resultados dinámicos que pueden ser plasmados en diversos formatos.

Si bien algunos autores definen un SIG como una base de datos espaciales, no es la principal función de estos softwares serlo. Una base de datos espaciales se circunscribe al modelado de datos y su almacenamiento, mientras que un SIG provee herramientas de análisis geográfico asi como de diseño y publicación cartográfica. La gama de funciones de estos programas es variable, según el segmento de usuarios al que van dirigidos, en este sentido Dueker (1987) y Cowen (1988) 
insisten en que lo más característico es su capacidad de análisis y de generar nueva información de un conjunto previo de datos mediante su transformación .

Hoy en dia muchos actores relacionados a la gestión del territorio utilizan los SIG para plasmar información en mapas temáticos de diversa índole, no obstante se puede observar que en su mayoria sus resultados son la expresión del uso de estos datos de un modo limitado a la simple expresión gráfica. Un ejemplo de ello, es que en muchos casos los SIG son utilizados como simples visualizadores de datos georreferenciados. Podriamos decir entonces que no presentan en muchos casos diferencias significativas de aquellas que podrian lograrse con un programa CAD o un programa de cartografía.

Si bien tanto en los SIG como en un programa CAD se trabaja con elementos gráficos (puntos, lineas, poligonos, y pixeles), solo en los primeros los elementos son relacionados a una base de datos espacial. Encontramos aquí la primera gran diferencia entre ambos sistemas, los CAD solo manejan los elementos gráficos de manera no relacional con otros datos. En este sentido, podemos decir por ejemplo que para un programa de $\mathrm{CAD}$ un polígono representa una longitud de perime tro y un área encerrada, incluso podemos definir las coordenadas de cada uno de sus vértices; pero si este polígono es representado en un SIG además llevará asociado una serie de datos y atributos referidos a ese mismo polígono.

Por otra parte, los SIG también presentan diferencias significativas con los programas de Cartografía asistida por ordenador, esto se debe fundamentalmente a que estos últimos no posibilitan el manejo de más de un conjunto de elementos gráficos al mismo tiempo.

Pero la capacidad más sobresaliente de los SIG es la de construir nuevos datos a partir de los ya existentes. Por tanto, un SIG es esencialmente diferente de un programa de dibujo (CAD), de un programa de gestión de bases de datos y de un programa de cartografía digital. No obstante se trata de una tecnología todavia inmadura por sus características complejas, que se traduce en el desconocimiento de sus potencialidades por quienes no son técnicos ni especialistas en la materia.

\section{Capacidades analíticas de los SIG;}

Dentro del ámbito de los SIG es posible crear consultas espaciales orientadas tanto a las entidades como a los atributos, e incluso obtener nuevos datos $y / o$ atributos a partir de la combinación de ambas generando situaciones reales o hipotéticas. A esta característica particular de los SIG se la denomina "Análisis espacial" o "geoprocesamiento".

Dentro de las capacidades de los SIG podemos distinguir tres aplicaciones que engloban sus funciones de análisis:

-Aquella que considera solo los datos desde un enfoque estadístico independiente de la componente espacial de los mismos.

-Aquella que considera solo la componente espacial de las entidades independiente de los datos asociados a ellas.

-Aquella que de modo integral considera ambos aspectos.

En términos generales la mayoría de los programas \$IG comparten las mismas funciones de análisis espacial, que permiten a los usuarios crear una serie de flujos de trabajo automatizados ayudando a realizar muchas de las tareas que de otra manera resultarían imposibles de concretar, como la conversión de datos, la verificación, la administración de coordenadas, y la producción de mapas entre otros.

Según diversos autores podemos sintetizar estas herramientas de procesamiento en:

-Interrogaciones: Se pueden utilizar criterios temáticos, espaciales y/o combinar ambos; las preguntas pueden ser simples o bien definidas dentro de parámetros concretos. Podemos realizar consultas sobre uno o más atributos de una o varias entidades, sobre varios atributos o entidades que se solapen, y que estén conectados o contenidos por otras entidades.

- Medidas: Estas funciones producen valores numéricos que describen algunas propiedades esenciales de los objetos, pero también nos permiten localizar entidades con respecto de otras según un criterio de localización. Sumarios. Son funciones que resumen los datos en unos cuantos indicadores. Los más usados son los de estadística descriptiva (media aritmética, mediana, moda, desviación típica, varianza) y sus equivalentes espaciales, como el centro de gravedad y la desviación típica de las distancias.

- Optimización: Se agrupan aquí diversas técnicas de naturaleza normativa cuya finalidad es la de seleccionar localizaciones que cumplen determi- 


\section{AT | Revista ARQUITECNO | N8}

nados criterios. También se incluyen los métodos de análisis de redes, como la búsqueda de rutas óptimas entre dos o más localizaciones en una red. -Transformaciones: Comprenden un conjunto de métodos simples de análisis espacial que transforman las entidades originales, mediante comparaciones o combinaciones. Estas funciones utilizan principios y reglas geométricas, aritméticas o lógicas, y también operadores de conversión de datos vectoriales. Dentro de estas últimas podemos encontrar además operaciones de:

-Reclasificación: Generan nuevos grupos de datos mediante la asignación de valores temáticos a las categorías existentes (tamaño, localización, forma, orientación, etc.)

Superposición: Producen nuevos mapas en los que el valor de cada elemento es el resultado de aplicar alguna función a los valores de los elementos de la misma localización de dos o más mapas

.Conectividad: Permiten evaluar la distancia entre objetos incorporando la fricción o resistencia del espacio al desplazamiento, así como la presencia de barreras y la naturaleza de las conexiones.

Vecindad: Consisten en procedimientos que asignan valores a una localización en función de los valores vecinos a ese punto.

\section{Objetivos:}

El presente trabajo tiene por objeto reflexionar sobre las potencialidades que presentan los $S I G$ en la gestión del territorio, cuando son utilizados como herramienta capaz de construir nuevos datos y nuevos elementos a partir de los ya existentes, en base a su análisis y procesamiento; considerando de modo integral los datos espaciales y sus atributos, lo que permite además aplicar técnicas de "modelado".

\section{Metodologia:}

El análisis espacial es el proceso de modelar, obtener resultados mediante funciones informáticas y luego examinar e interpretar los resultados del modelo. Con él, se puede combinar información de numerosos orígenes independientes y obtener un grupo completamente nuevo de información (resultados) mediante la aplicación de un conjunto de operadores espaciales. En el proceso de análisis espacial o geoprocesamiento se aplican técnicas analíticas referenciadas geográficamente para extraer o generar nueva información para tratar una cuestión o un objetivo en particular. Este proceso en general e independientemente del software que se utilice comprende los mismos pasos.

Establecer un objetivo y formular las preguntas que se desea responder.

-Reunir, organizar y preparar los datos para el análisis.

- Crear el modelo de análisis (proceso que generalmente se realiza mediante geoprocesamiento).

-Ejecutar el modelo y generar resultados.

-Explorar, evaluar, resumir, interpretar y analizar los resultados.

-Representar y visualizar los resultados.

El proceso de análisis consiste por una parte en crear un modelo basado en los objetivos de análisis y por otra en generar una serie de mapas, informes, gráficos, y resúmenes del análisis. En cada paso se realiza una revisión (los resultados se representan cartográficamente, se comparan, visualizan, interpretan, modifican, actualizan, se vuelven a ejecutar, etc.) que ofrece la oportunidad de incorporar nuevos conocimientos adquiridos durante el proceso. Para finalizar, se exploran e interpretan los resultados y se los utilizan para sacar conclusiones y tomar decisiones.

\section{Caso de aplicación:}

Se toma como ejemplo para este trabajo, el empleo del SIG como herramienta en la simulación de escenarios de riesgo sísmico en el área metropolitana de la ciudad de San Juan (AM GSJ), Argentina. En este caso se utilizaron herramientas de análisis estadístico y espacial, construyendo índices probabilisticos de riesgo, mediante la combinación de diferentes capas temáticas que representan las diferentes variables.

A efectos de determinar la vulnerabilidad sismica del área de referencia se utilizó, en el marco de un proyecto de investigación ${ }^{1}$, un método que permitió identificar el estado de daños probable en cada una de las edificaciones contenidas en las parcelas.

Inicialmente se construyó un mapa de amenazas que incluyo la superposición de diversas variables de análisis entre las que podemos citar la ubicación y dirección de fallas sismicas, las curvas de aceleración sismicas, el área de ribera del rio San Juan, las zonas de napas freáticas entre 0.5 y $2.0 \mathrm{~m}$ de profundidad, los suelos con mayor peligrosidad de licuefacción, los niveles de contaminación y nitratos de los suelos, las áreas en las que las viviendas presentan pozos absorbentes para desagües, y los vertederos de residuos y escombreras 
(ver figura No 1).

Por otro lado se construyó un mapa producto de la combinación de las características de las construcciones en función del tipo edilicio (destino de la construcción), el estado de mantenimiento de las mismas, el año en que fueron construidas que responde a su vez a los criterios de diseño sísmicos utilizados, y la altura de la edificación. Todos estos datos combinados mediante la aplicación de herramientas de análisis espacial "Interrogación - Transformación -Superposición" generaron nuevos datos que representaban un índice de daños en función de un sismo de determinada intensidad que fueron representados estadisticamente y en gráficos 2 y $3 \mathrm{D}$ (ver figura No 2 y 3 ).

No obstante, al momento de graficar las caracteristicas de cada polígono (edificación) resultó dificultosa la visualización en las escalas de impresión (ver figura No 4), por lo que se decidió asignarle a la parcela las características de la edificación de mayor superficie aplicando herramientas de "Transformación - Reclasificación". De esta manera, los datos de las edificaciones quedan graficados a escala de parcelas, permitiendo una mejor y clara lectura (ver figura No 5).
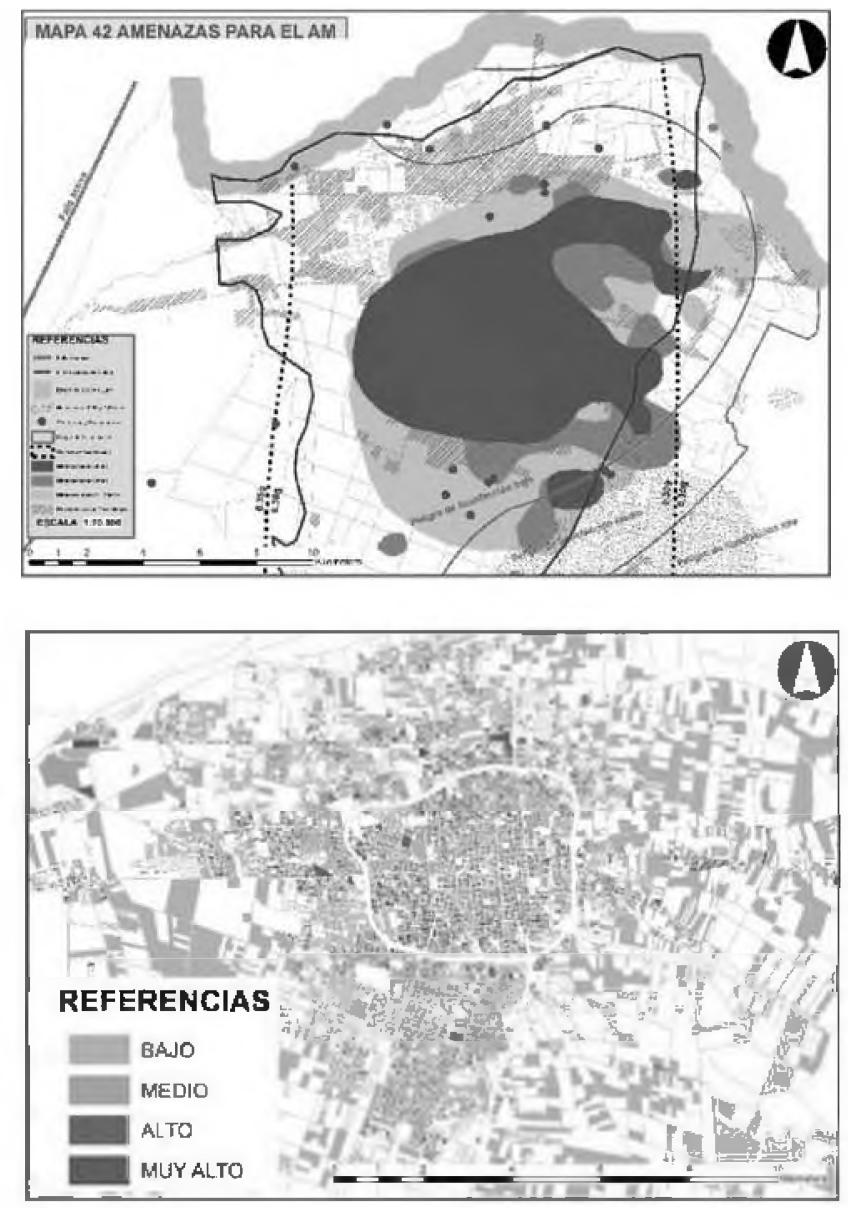

Figura 1: Mapa de amenazas para el AMS

Figura 2: Indice de vulnerabilidad fisica de las construcciones.

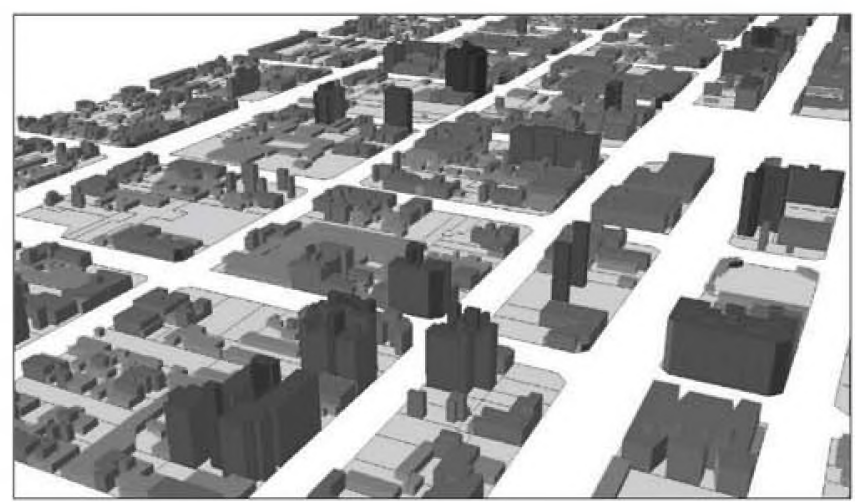

Figura 3: lndice de wulnerabilidad fisica de las construcciones modelado en 3D

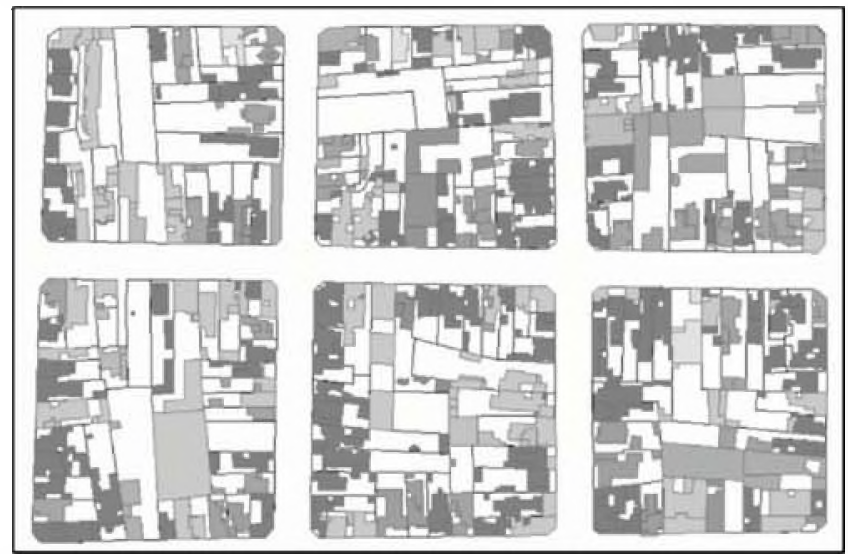

Figura 4: Representación a escala de edificación.

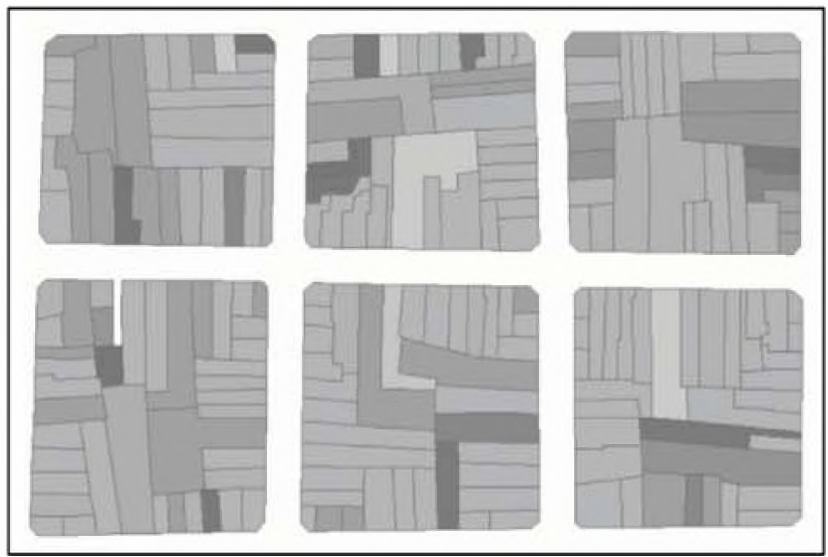

Figura 5: Representación a escala de parcelas.

Por otro lado también se observó la existencia de grandes parcelas con construcciones pequeñas precarias. En este caso al asignarle a la parcela las características de la edificación se pueden generar grandes áreas con características de vulnerabilidad sísmica que no son reales. Para ello cuando esta última supere el $25 \%$ de la superficie de la parcela aplicando técnicas de "Interrogación - Medidas - Transformación".

Todos estos datos al ser integrados en un SIG pudieron además ser representados en diversos formatos que permitieron no solo el análisis espacial sino además un análisis estadístico a través de las herramientas de "Sumarios" como puede observarse en la figura No6. 
AT | Revista ARQUITECNO | N8

\begin{tabular}{|c|c|c|c|c|c|c|}
\hline PEFUODO & \multicolumn{2}{|c|}{ EDAD } & \multicolumn{2}{|c|}{ CODHGO } & \multicolumn{2}{|c|}{ FACTOA } \\
\hline 19ta - 2009 & \multicolumn{2}{|c|}{$\therefore 21$ anos } & \multicolumn{2}{|c|}{ FRES CFSOC 103} & \multicolumn{2}{|r|}{18} \\
\hline 49t0 = 19:7 & \multicolumn{2}{|c|}{22 a 2 a atos } & \multicolumn{2}{|l|}{ Aa a } & \multicolumn{2}{|c|}{$0,0 \%$} \\
\hline $1970-1979$ & \multicolumn{2}{|c|}{30 ด 39 arios } & \multicolumn{2}{|l|}{ CNCAR 70} & \multicolumn{2}{|c|}{$0,00 x$} \\
\hline $1946-1969$ & \multicolumn{2}{|c|}{ AOS EAt trios } & \multicolumn{2}{|c|}{ DOCHOO DE EDFMCACION } & \multicolumn{2}{|c|}{$0.50 \%$} \\
\hline Antes 1944 & \multicolumn{2}{|c|}{35 whos } & \multicolumn{2}{|l|}{$\cos \cos 0$} & \multicolumn{2}{|c|}{$0.7 \%$} \\
\hline DEPARTAMERTO & FRACEION & RADOO & SEGMENTO & MANZUNA & PARCELA & EDIFICIO \\
\hline Capital & 7 & 28 & 39582 & 117 & 33521 & 80479 \\
\hline Chimbas & 3 & 27 & 17842 & 498 & $188 \mathrm{X} 7$ & 31150 \\
\hline Pocita & 4 & 16 & 2054 & 305 & 11659 & 11598 \\
\hline Rawson & 6 & 29 & 28350 & 912 & 26457 & 51276 \\
\hline Ruvadavia & 5 & 28 & 20761 & 571 & 22009 & 35827 \\
\hline Santa Lucia & 4 & 19 & 12321 & 337 & 13478 & 23530 \\
\hline
\end{tabular}

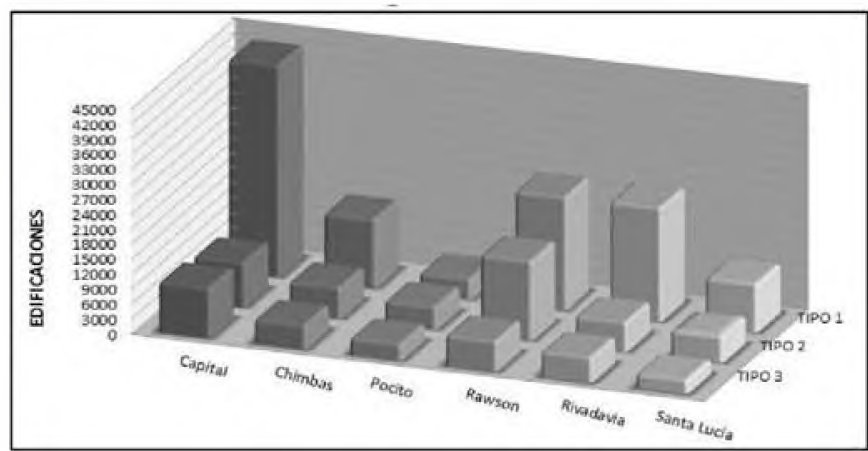

Figuras 6: Representaciỏn de la construcción según TIPO.

En cuanto a la vulnerabilidad social, se trabajó con datos censales desagregados por fracción y radio. Se creó un Índice socio-habitacional ${ }^{2}$, que sintetiza la situación de la educación, el estado de la cobertura y el empleo de la salud de la población, necesidades básicas y la calidad de construcción y materiales. Esta medida resumen producto nuevamente del uso de herramientas de reprocesamiento "Transformación Reclasificación" permitió la identificación de áreas con características similares, datos que también pudieron ser representados geográfica y estadisticamente (ver figura No 7).
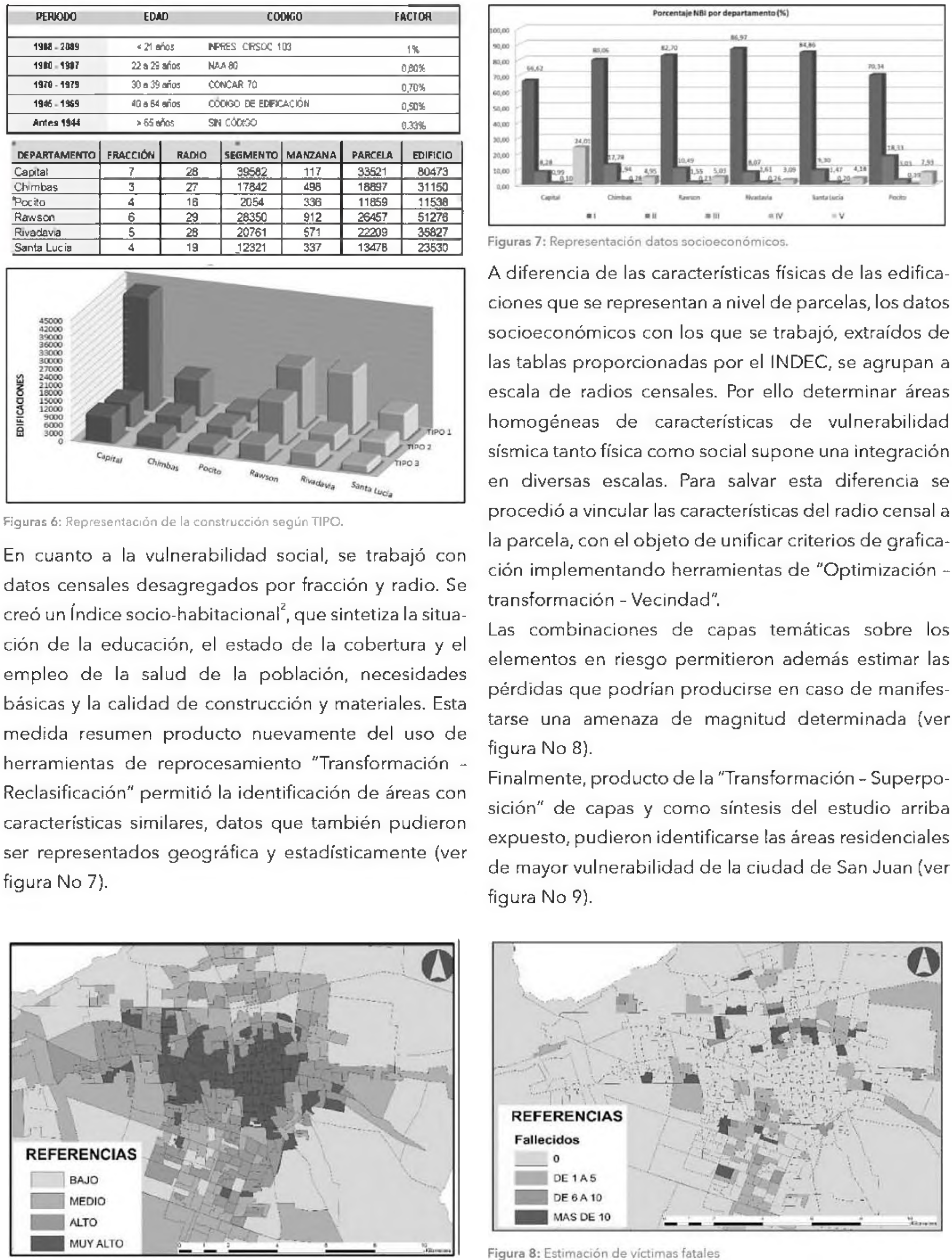

Figuras 7: Representación datos socioeconómicos.

A diferencia de las características físicas de las edificaciones que se representan a nivel de parcelas, los datos socioeconómicos con los que se trabajó, extraídos de las tablas proporcionadas por el INDEC, se agrupan a escala de radios censales. Por ello determinar áreas homogéneas de características de vulnerabilidad sismica tanto fisica como social supone una integración en diversas escalas. Para salvar esta diferencia se procedió a vincular las características del radio censal a la parcela, con el objeto de unificar criterios de graficación implementando herramientas de "Optimización . transformación - Vecindad".

Las combinaciones de capas temáticas sobre los elementos en riesgo permitieron además estimar las pérdidas que podrian producirse en caso de manifestarse una amenaza de magnitud determinada (ver figura No 8).

Finalmente, producto de la "Transformación - Superposición" de capas y como sintesis del estudio arriba expuesto, pudieron identificarse las áreas residenciales de mayor vulnerabilidad de la ciudad de San Juan (ver figura No 9).

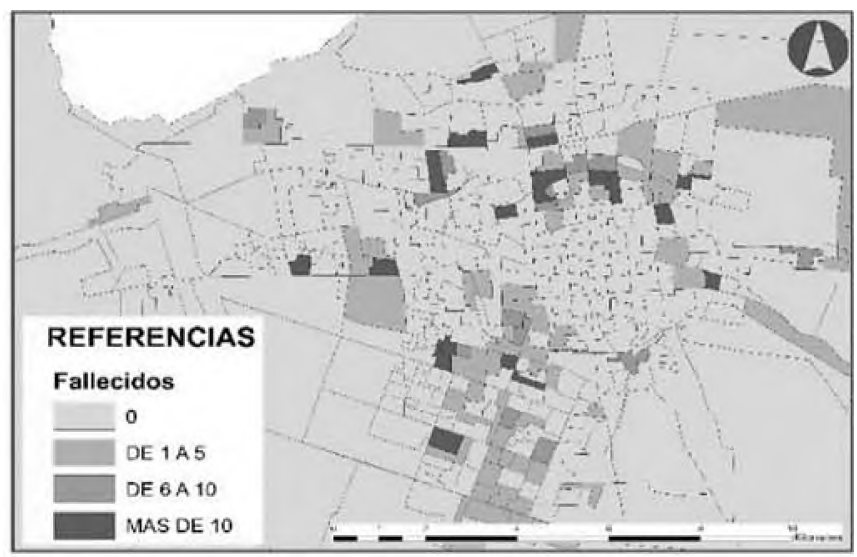

Figura 8: Estimación de victimas fatales 


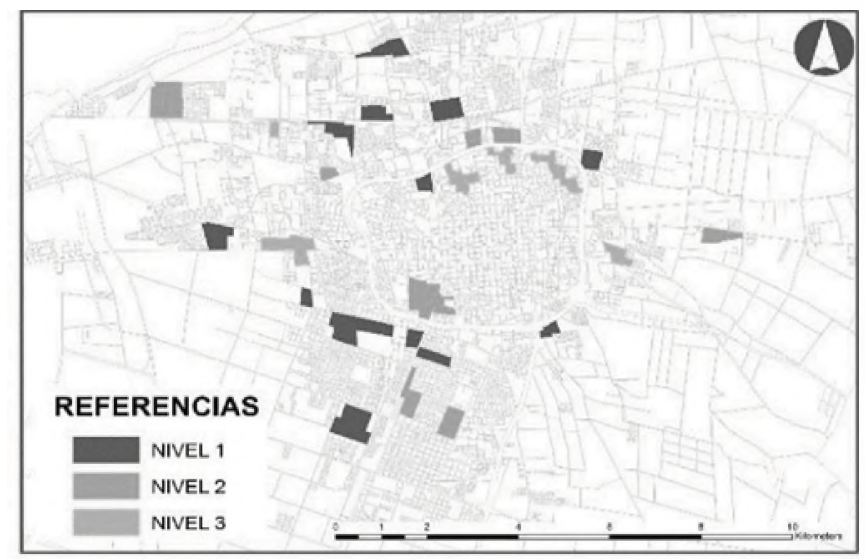

Figura 9: Areas vulnerables del AMSJ.

\section{Conclusiones:}

Como expresamos inicialmente en muchos casos los SIG son utilizados como simples visualizadores de datos georreferenciados, $\sin$ presentar diferencias significativas de aquellas que podrían lograrse con un programa CAD o un programa de cartografía digital. Si bien los programas que trabajan en ámbitos $\mathrm{CAD}$ son capaces de superar las limitaciones de contenidos que los mapas tradicionales tienen, otorgando calidad de representación y precisión posicional de los elementos que representan, en el análisis espacial siguen siendo insuficientes en comparación con los programas SIG. Podemos entonces inferir, luego de analizar el ejemplo aquí expresado, que cuando los SIG son implementados en consecuencia con sus reales potencialidades presentan ventajas considerables en relación a que:

- Los Sistemas de Información Geográficos, al permitir relacionar datos gráficos (planos o mapas urbanos) con otros datos a atributos alfanuméricos (demográficos, geológicos, características de la edificación, etc.) que pueden ser permanentemente actualizados, constituyen una herramienta imprescindible en la gestión del territorio. La implementación de esta tecnología permite suponer diversos escenarios y plantear acciones en función de diversas variables.

-Facilitan asimismo el manejo de volúmenes considerables de información, generando su entrecruzamiento en condiciones controladas, lo que hace posible la rápida evaluación de distintas situaciones. Esta rapidez y flexibilidad aparecen como la mayor ventaja de la utilización de estos sistemas.

-Además, la implementación de los SIG reduce las probabilidades de duplicar tanto la información como el trabajo, acortando los tiempos de ejecución, duplicando la productividad y permitiendo compartir datos de manera rápida y sencilla entre distintas áreas destinadas a abordar el problema, lo que además facilita la toma de decisiones y mejora la coordinación.

-Presentan una rápida producción de mapas e informes en comparación con el tiempo empleado manualmente, mejorando la amplitud y profundidad de los análisis y facilitando además la evaluación de diferentes estrategias simultáneamente.

-Por último, mejoran la capacidad de actualización de la información permitiendo la automatización de estos procesos generando información reciente de cada variable.

\section{Referencias:}

-BOSQUE SENDRA y otros. (2000) "El uso de los sistemas de información geográfica en la planificación territorial" Publicado en Anales de Geografía de la Universidad complutense, no 20, pp. 49-67

MASKREY Andrew (1998) "Navegando Entre Brumas La aplicación de los Sistemas de Información Geográfica al Análisis del Riesgo en América Latina". ITDG/La Red, Colombia ISBN 9972-47-017-2.

-PEÑA LLOPIS Juan (2006) "Sistemas de Información Geográfica Aplicados a la Gestión del Territorio" Club Universitario., España ISBN 84-8454-493-1

-ROMERO Mirta y otros. (2004). Metodología de estimación de daños a la edificación producidos por terremotos, mediante el empleo de los SIG. Revista PROAMBIENTE del Programa de Estudios Ambientales de la U.N.S.J. Volumen 3 - Págs. 1 a 10. San Juan, Argentina.

-ROMERO Mirta y otros. (2005) "El uso de Modelos digitales en la Planificación Urbana". Ciudad y Territorio Virtual. Revista del Laboratorio de Estudios Urbanos de la Universidad de Bio ISBN 956-7813-38-8. 